\title{
Correlations of quantitative MRI metrics with Myelin Basic Protein (MBP) staining in a murine model of demyelination
}

\author{
Lucas Soustelle $^{1}$ | Maria C. Antal ${ }^{1}$ | Julien Lamy ${ }^{1}$ | François Rousseau ${ }^{2}$ | Jean-Paul \\ Armspach $^{1}$ | Paulo Loureiro de Sousa ${ }^{1}$
}

${ }^{1}$ Université de Strasbourg, CNRS, ICube, FMTS, Strasbourg, France

${ }^{2}$ Institut Mines Télécom Atlantique, INSERM, LaTIM, Brest, France

\section{Correspondence}

Lucas Soustelle, CRMBM, UMR 7339 -

Aix-Marseille Université, Faculté de médecine,

27 Boulevard Jean Moulin, 13389 Marseille,

France.

Email: lucas.soustelle@gmail.com

\begin{abstract}
Summary
Myelin imaging in the central nervous system is essential for monitoring pathologies involving white matter alterations. Various quantitative MRI protocols relying on the modeling of the interactions of water protons with myelinated tissues have shown sensitivities in case of myelin disruption. Some extracted model parameters are more sensitive to demyelination, such as the bound pool fraction (f) in quantitative magnetization transfer imaging (qMTI), the radial diffusivity in diffusion tensor imaging (DTI), and the myelin water fraction (MWF) in myelin water imaging (MWI). A 3D ultrashort echo time (UTE) sequence within an appropriate water suppression condition (Diff-UTE) is also considered for the direct visualization of the myelin semi-solid matrix (Diff-UTE normalized signal; rSPF). In this paper, we aimed at assessing the sensitivities and correlations of the parameters mentioned above to an immuno-histological study of the Myelin Basic Protein (MBP) in a murine model of demyelination at $7 \mathrm{~T}$. We demonstrated a high sensitivity of the MRI metrics to demyelination, and strong Spearman correlations in the corpus callosum between histology, macromolecular proton fraction $(\rho>0.87)$ and Diff-UTE signal $(\rho>0.76)$, but moderate ones with radial diffusivity and MWF $(|\rho|<0.70)$.
\end{abstract}

KEYWORDS:

UTE imaging, quantitative magnetization transfer, myelin water imaging, diffusion tensor imaging, demyelination, histology, Myelin Basic Protein

\section{Word count: 4521}

\section{1 | INTRODUCTION}

Assessment of myelin content in the brain is essential for monitoring pathologies such as multiple sclerosis. Quantitative MRI methods such as diffusion tensor imaging (DTI) 1 , myelin water imaging (MWI ${ }^{2}$ and quantitative magnetization transfer imaging (qMTI) ${ }^{3}$ are often employed in animal and human studies to assess demyelination processes, although analyses can be affected by other factors such as inflammation and gliosis 456 . Radial diffusivity $\left(\lambda_{\perp}\right)$ in DTI is sensitive to myelin changes 78 , in addition to other factors such as axon diameter and crossing fiber bundles ${ }^{9}$. qMTI probes macromolecular content by indirectly assessing exchanges between bound and free proton pools 10 . The bound pool fraction ( $f$ ), a metric derived from qMT, is sensitive to myelin content, but is also influenced by sequence-dependent parameters ${ }^{11}$. MWI evaluates the proton fraction attributed to the trapped water between the myelin bilayers (MWF) which demonstrates shorter $\mathrm{T}_{2}$ values than intra/extra-axonal water 12 . The

${ }^{0}$ Abbreviations: UTE, ultrashort echo time; qMTI, quantitative magnetization transfer imaging; MWI, myelin water imaging; DTI, diffusion tensor imaging; MBP, Myelin Basic Protein; rSPF, relative Semi-solid Proton Fraction 
original technique (multi-spin-echo based) has been improved by compensating for the stimulated echo effects during the post-processing estimation 1314 . Alternatively, multi-gradient echo MWI techniques were proposed to compensate for spin-echo based issues 15,1617 . Deoni et al. 18 proposed a quantification of the three main water pools using a more sophisticated biophysical model taking into account inter-compartments exchanges. However, regardless of the model, the MWI techniques still suffer from a lack of consensus and precision 192021 . Additionally, a preclinical study demonstrated the moderate specificity of the method in case of inflammation using a histological analysis 22 .

All of these state of the art methods for myelin imaging and quantification exploit chemical or magnetic interactions of the water with the myelin semi-solid part. Hence, the specificity and the selectivity of these techniques may vary with their respective models and hypotheses. Other related issues may occur with various clinical MRI considerations (e.g. protocol duration or specific absorption rate).

Protons from the macromolecular matrix of myelin (lipids and proteins) in the central nervous system demonstrate rather short $\mathrm{T}_{2}$ values because of intense and unaveraged dipolar coupling in these solid-like structures 23 . Several studies suggest that myelin can be directly visualized with ultrashort echo time (UTE) sequences, hence potentially overcoming the lack of specificity of other indirect methods. Horch et al. ${ }^{24}$ and Wilhelm et al. ${ }^{25}$ spectroscopically explored myelinated tissue samples, demonstrating the presence of a wide distribution of sub-millisecond $\mathrm{T}_{2}$ values, as well as a high correlation between the myelin concentration and corresponding integrated ${ }^{1} \mathrm{H}$ spectra. UTE techniques remain somehow challenging as they require an appropriate suppression of long- $T_{2}$ components $\left(T_{2}>10 \mathrm{~ms}\right)$ in order to generate a selective short- $T_{2}\left(T_{2}<1 \mathrm{~ms}\right)$ contrast. Many efforts were led in human studies using an Inversion-Recovery (IR) based UTE sequence, although in a single slice 2D fashion 262728. Additionally, the use of an adiabatic inversion pulse in the IR module for homogeneous long- $T_{2}$ suppression purpose involves a short- $T_{2}$ signal attenuation 2930 . A 3D UTE sequence performing an appropriate water suppression condition through diffusion weighting (Diff-UTE) was previously proposed to compensate for these limitations 31 , providing high contrast in myelinated areas of an ex vivo healthy mouse brain 32 .

Several MRI quantitative metrics extracted from the techniques mentioned above are often employed as biomarkers of demyelination, but their respective specificities vary according to the investigated myelin disorder. In this study, we assess the specificity to demyelination of several quantitative MRI parameters at 7T by comparison to the fluorescence yield of a myelin marker (myelin basic protein - MBP) in normal and demyelinized ex vivo mouse brains 4533 .

\section{2 | MATERIALS AND METHODS}

\section{1 | Animal experiments}

Animal studies were conducted in agreement with the European Council Directive 2010/63/EU and the French Guidelines for Animal Care from the French Department of Agriculture (Animal Rights Division), and approved by our institutional committee on ethics in animal research (CREMEAS $\mathrm{AL} / 41 / 48 / 02 / 13$ ). Female C57BL/6 mice (8 healthy vs. 7 cuprizone-fed with a respective mean weight of $20.9 \pm 1.8 \mathrm{~g}$ and $16.4 \pm 1.2 \mathrm{~g}$ ) were sacrificed at 13 weeks of age. Demyelination was induced by cuprizone administration in standard chow (0.2\%) for 5 consecutive weeks prior to sacrifice. Mice were transcardially perfused $(2.0 \mathrm{~mL} / \mathrm{min}$ ) with $4 \%$ paraformaldehyde (PFA; nominal duration: $10 \mathrm{~min})$ under deep anesthesia, preceded by heparinized physiological serum and followed by phosphate buffered saline solution (PBS) flushing (nominal durations: 4 min). Samples were stored at $4^{\circ} \mathrm{C}$ in PBS for at least 15 days for tissue stabilization. Perfusion durations were adapted to the respective weight of the mouse, with a nominal duration of $10 \mathrm{~min}$ for $20 \mathrm{~g}$. For scanning, fixed mouse heads were soaked into PFPE (Galden, Solvay) before scanning at room temperature $\left(\approx 20^{\circ} \mathrm{C}\right)$.

\subsection{Quantitative MRI protocols and analyses}

All mice were scanned on a 7T preclinical scanner (Bruker BioSpec, Ettlingen, Germany), using a 23-mm volume Tx/Rx coil in order to provide an homogeneous $B_{1}$ field in the slab of interest. All 3D MRI datasets were acquired using the same axial volume with 0.750-mm thick slices.

\subsection{1 $\mathrm{DTI}$, qMTI and MWI quantitative protocols}

DTI, qMTI and MWI protocols shared the same slice geometry $\left(100 \times 100 \times 750 \mu \mathrm{m}^{3}\right)$.

The relevant parameters were: 1 ) DTI (Pulsed Gradient Spin-Echo): matrix size $=128 \times 128 \times 24$, TR $/$ TE $=750 / 27 \mathrm{~ms}$, single-shell 7-directions with a low b-value $18.9 \mathrm{~s} / \mathrm{mm}^{2}$ and a high one of about $1000.0 \mathrm{~s} / \mathrm{mm}^{2}, \delta / \Delta=6 / 14 \mathrm{~ms}$ ( $\mathrm{T}_{\mathrm{acq}}=307 \mathrm{~min}$ ); 2 ) MWI (Multi Spin-Echo): matrix size $\left.=128 \times 128 \times 24, \mathrm{TR} / \mathrm{TE} / \mathrm{ESP}=2000 / 10 / 10 \mathrm{~ms}\left(\mathrm{~T}_{\mathrm{acq}}=102 \mathrm{~min}\right) ; 3\right) \mathrm{qMTI}(\mathrm{MT}-\mathrm{SPGR}):$ matrix size $=128 \times 128 \times 32, \mathrm{TR} / \mathrm{TE}=70 / 3 \mathrm{~ms}$, frequency offsets $=1 / 2 / 4 / 6 / 10 / 30 \mathrm{kHz}, 267-\mathrm{Hz}$ wide gaussian saturation pulse $\left(\tau_{\text {sat }}=10.25 \mathrm{~ms}\right)$ with $\mathrm{B}_{1}^{\text {peak }}=5 / 10 / 20 \mu \mathrm{T}$, excitation flip angle $=22^{\circ}, r B W=50 \mathrm{kHz}, 2$ signal acquisitions for $\mathrm{MT}_{0}\left(\mathrm{~T}_{\mathrm{acq}}=215 \mathrm{~min}\right)$. Additionally, $\mathrm{B}_{1}, \mathrm{~B}_{0}$ and $\mathrm{T}_{1}$ corrections were provided in the qMTI protocol, using the following parameters: 4) $B_{1}$ (Actual Flip angle Imaging (AFI) SPGR): matrix size $=48 \times 48 \times 48\left(\right.$ voxel size $=250 \times 250 \times 750 \mu \mathrm{m}^{3}$ ), 


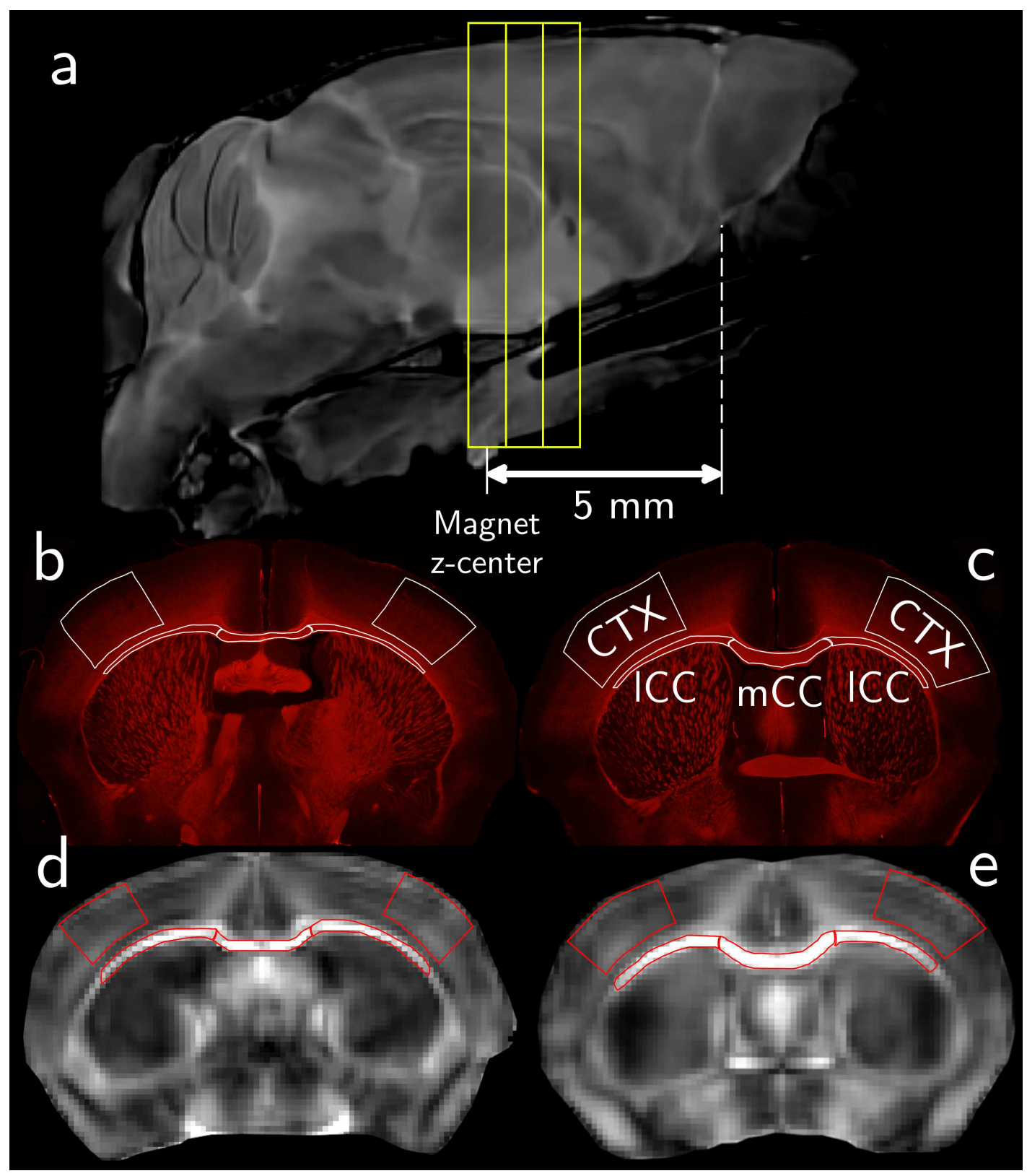

FIGURE $13 \mathrm{D}$ sub-volume positioning based on a high-resolution $\mathrm{T}_{2}$-weighted sagittal image (a), and corresponding ROIs selected in the somatosensory cortex (CTX) and corpus callosum (ICC and $\mathrm{mCC}$ ) in a caudal (b) and rostral (c) MPB immuno-histological views, as well as on a representative fractional anisotropy map (d-e). Mice heads were positioned so that the corpus callosum was perpendicular to the slice plane in order to limit partial volume effects.

$\mathrm{TR}_{1} / \mathrm{TR}_{2} / \mathrm{TE}=15 / 60 / 2 \mathrm{~ms}$ and nominal flip angle $=60^{\circ}, \mathrm{rBW}=50 \mathrm{kHz}, 4$ signal acquisitions ( $\left.\left.\mathrm{T}_{\text {acq }}=11 \mathrm{~min}\right) \stackrel{34}{34}^{5}\right) \mathrm{B}_{0}$ (dual-echo SPGR): matrix size $=48 \times 48 \times 48\left(\right.$ voxel size $\left.=250 \times 250 \times 750 \mu \mathrm{m}^{3}\right), \mathrm{TR} / \mathrm{TE}_{1} / \mathrm{TE}_{2}=20 / 1.65 / 3.55 \mathrm{~ms}$, flip angle $=30^{\circ}, \mathrm{rBW}=119 \mathrm{kHz}, 2 \mathrm{signal}$ acquisitions, acquired prior to each $B_{1}^{\text {peak }}$ series $\left.\left(\mathrm{T}_{\text {acq }}=24 \mathrm{~min}\right) ; 6\right) \mathrm{T}_{1}$ (Variable Flip Angle SPGR): matrix size $=128 \times 128 \times 32\left(\right.$ voxel size $\left.=100 \times 100 \times 750 \mu \mathrm{m}^{3}\right)$, $\mathrm{TR} / \mathrm{TE}=100 / 2.7 \mathrm{~ms}$, flip angles $=10 / 25 / 40 / 80^{\circ}, 3$ signal acquisitions $\left(\mathrm{T}_{\mathrm{acq}}=82 \mathrm{~min}\right)$.

The total acquisition duration amounted to $12 \mathrm{~h} 37 \mathrm{~min}$. In SPGR-like sequences, a strong gradient spoiling was systematically included to crush the remaining transverse magnetization through diffusion effects, along with an appropriate RF spoiling (SPGR: $\phi_{0}=117^{\circ}$ [35, AFI-SPGR: $\phi_{0}=129.3^{\circ}$ using Nehrke's spoiling scheme ${ }^{36}$ ). 


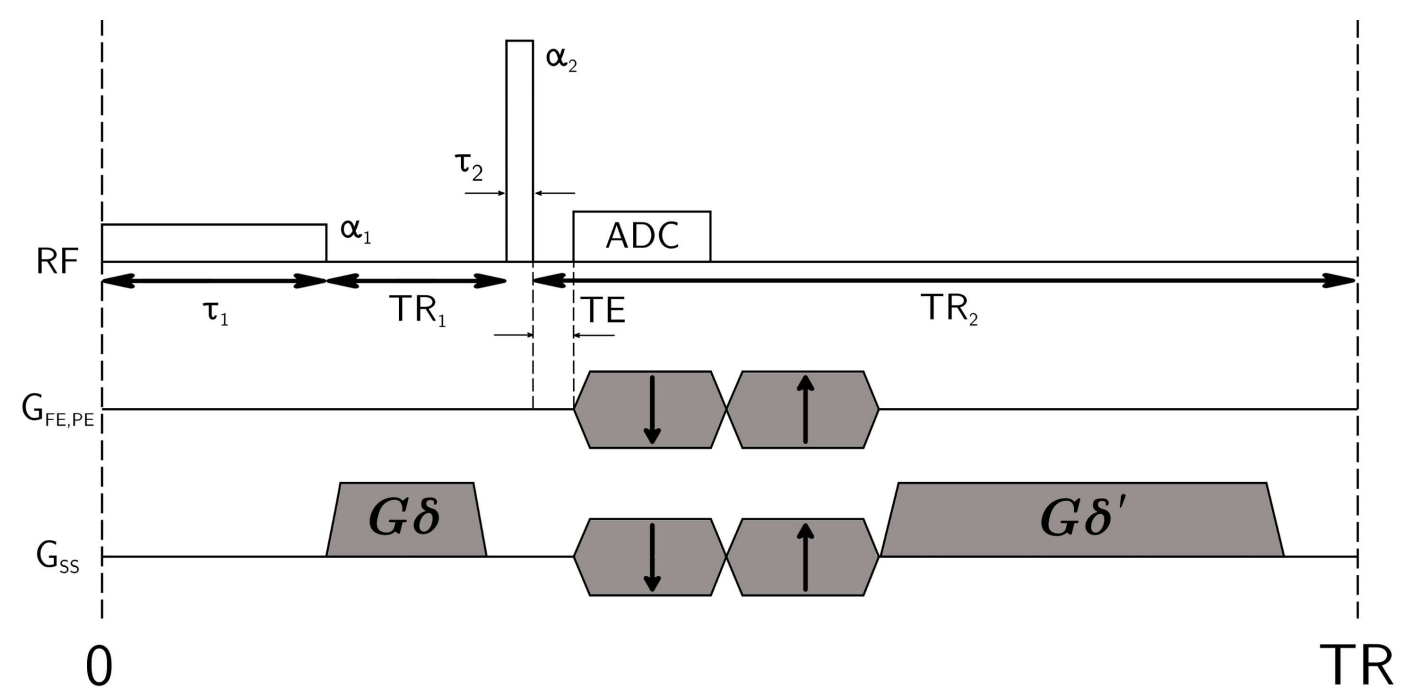

FIGURE 2 Diff-UTE pulse sequence. The gradients inducing diffusion weighting in Diff-UTE correspond respectively to the short one after the preparation pulse (relative area $\mathrm{G} \delta$ ), and to the long one after the readout gradients refocusing (relative area $\mathrm{G} \delta^{\prime}$ ).

\subsection{2 | Diff-UTE protocol}

The Diff-UTE sequence (depicted in Figure 2 was previously proposed as a 3D-UTE method allowing an efficient long-T 2 suppression condition in biological tissues while maximizing the signal of a short- $\mathrm{T}_{2}$ component ${ }^{32,31}$. This hard-pulse prepared sequence takes advantage of the particular magnetization steady-state condition to generate a signal cancellation of a diffusive and long-relaxing component through a diffusion weighting induced by spoiling gradients. After generation of the optimized parameters maximizing the short- $\mathrm{T}_{2}$ component through the Bloch equations given priors about the myelin relaxing times $\left(T_{1} / T_{2} \approx 230 / 0.1 \mathrm{~ms}\right)$, the areas of the spoiling gradients are tuned to provide an appropriate signal nulling of the diffusive long- $T_{2}$ component of the white matter of an ex vivo healthy mouse $\left(T_{1} / T_{2} \approx 1300 / 67\right.$ ms using respectively an inversionrecovery spin-echo sequence and a multi-spin echo sequence, and ADC $\approx 0.328 \mu \mathrm{m}^{2} / \mathrm{ms}$ using a pulse gradient spin echo sequence with diffusion weighting along the same spoiling direction in Diff-UTE). Previous studies demonstrated the efficiency of signal cancellation through this process 32 .

In this sequence, the following optimized parameters were used: matrix size $=84 \times 84 \times 84$ ( 21938 radial projections, 50 points $/$ spoke), voxel size $=152 \times 152 \times 750 \mu \mathrm{m}^{3}, \mathrm{TR}_{1} / \mathrm{TR}_{2}=5 / 25 \mathrm{~ms}, \tau_{1} / \tau_{2}=1.0 / 0.01 \mathrm{~ms}, \alpha_{1} / \alpha_{2}=90 / 50^{\circ}, \mathrm{G} \delta=198.9 \mathrm{mT} / \mathrm{m} . \mathrm{ms}$, RF phase increment $\phi_{0}=0^{\circ}$, receiver bandwidth $(\mathrm{rBW})=150 \mathrm{kHz}, 12$ signal acquisitions $\left(\mathrm{T}_{\mathrm{acq}}=136 \mathrm{~min}\right)$. The Diff-UTE signal was normalized to that estimated in a piece of rubber $\left(T_{1} / T_{2}^{*} \approx 182 / 0.3 \mathrm{~ms}\right.$ at $\left.3 T^{[27}\right)$ spatially localized in the same rostral position throughout the scans. The signals ratio is referred hereafter to as relative semi-solid proton fraction (rSPF).

\subsection{3 | MR quantitative image analysis}

Before parameters estimations, and to compensate for potential $\mathrm{B}_{0}$-drift effects inducing FOV shifting, all volumes were rigidly registered onto the lowest b-value image from the DTI protocol using the Advanced Normalization Tools (ANTS) registration package 37 , 38, followed by a denoising step using Block-matching and 3D filtering (BM4D) ${ }^{39}$. Due to its unconventional contrast, the automatic registration of the Diff-UTE volume could not be performed. Hence, it was manually achieved using the registration tool from ITK-Snap onto the highly-contrasted Fractional Anisotropy (FA) map from DTI.

For quantitative purpose, $B_{0}$ maps were estimated using the manufacturer algorithm in PV6.0.1, and all other maps were reconstructed using a home-made pipeline, implemented on Matlab 2016a (The MathWorks, Inc., Natick, MA, USA). $B_{1}$ map was estimated using the AFI method ${ }^{34}$. In DTI, FA and $\lambda_{\perp}$ were estimated using a constrained non-linear least-square fitting process with a Cholesky decomposition correction 4 . In qMTI, $f$ was estimated using Sled and Pike's rectangular pulse (RP) model implemented in the qMTLab toolbox ${ }^{40}$, with equal relaxation rates between the restricted and free pools $R_{1, r}=R_{1, f}$. $B_{1}, B_{0}$ and $T_{1}$ values were provided, and the transverse relaxation constant of the restricted pool was fixed to $10 \mu \mathrm{s}$ for the entire dataset as it demonstrates a narrow range in tissues 414 . MWF was estimated based on a home-made Non-Negative Least Square (NNLS) framework ${ }^{2}$, taking account of potential stimulated echoes effects ${ }^{13}$. The $\mathrm{T}_{2}$ spectra comprised $40 \mathrm{~T}_{2}$ values logarithmically spaced from 15 to $1500 \mathrm{~ms}$. To overcome the ill-conditioned issues of this inverse problem, a Tikhonov regularization was performed (rNNLS), and results were further smoothed by introducing a spatial prior (srNNLS) in the optimization process based on the spectra estimated in the rNNLS maps $\frac{42}{\text {. }}$ 
For MWF maps computation, the cutoff of the estimated spectra was empirically set to $35 \mathrm{~ms}$ based on prior analyses in regions of interest in the corpus callosum of the healthy mice.

\subsubsection{Immuno-histological MBP staining and fluorescence quantification}

After ex vivo imaging, mice brains were extracted and soaked into a $20 \% \mathrm{w} / \mathrm{v}$ sucrose solution between 24 and 48 hours, prior to freezing and storage at $-20^{\circ} \mathrm{C}$. Following overnight thawing at $4{ }^{\circ} \mathrm{C}, 60-\mu \mathrm{m}$ coronal sections were collected within a 3 mm-thick volume starting at the optic chiasm. The size of the histological initial block was chosen to encompass the slices used for MRI analyses. Sections were then soaked into $20 \%$ sucrose solution prior to freezing and storing at $-20^{\circ} \mathrm{C}$.

After thawing, two sections were selected for MPB staining. The first section was localized $1 \mathrm{~mm}$ rostral to the bregma, the second one $1 \mathrm{~mm}$ caudal to the bregma, in order to account for the different susceptibility to demyelination along the corpus callosum 4344 . The following staining protocol was used: i) washing in PBS at $4^{\circ} \mathrm{C}$ for $12 \mathrm{~h}$; ii) washing in PBS-Triton (0.1\%) at room temperature for 15 min; iii) incubation with a homemade mouse antibody anti-MBP at room temperature for $24 \mathrm{~h}$ under agitation; iv) washing in PBS-Triton (0.1\%) at room temperature for 15 min; $v$ ) incubation with an anti-mouse Alexa-594 antibody (1/500; Thermo Fisher Scientific, Waltham, MA) at room temperature for $24 \mathrm{~h}$ under agitation; vi) washing in PBS-Triton (0.1\%) at room temperature for $15 \mathrm{~min}$; vii) washing in PBS at room temperature for 15 min.

Sections were then mounted on glass slides (Dako; Agilent Technologies, Santa Clara, CA) and examined using an epifluorescence microscope (DP70, magnification x2; Olympus, Tokyo, Japan). Photographs were taken under constant gain and exposure duration (1.1 s) parameters. For quantitative, the Mean Fluorescent Intensities (MFI) of the red channel were estimated from the same regions of interest (ROI) as the MRI ones. The mean background intensity estimated from a ROI far from tissues was subtracted from the signal to compensate for any non-signal contribution.

\subsection{5 | Regions of interest selection}

The ROIs were drawn twice: in histology over the MPB views, and in MRI over the high-contrast fractional anisotropy map estimated in DTI. To limit potential user-dependent selection of out-of-ROI voxels during delineation, parameter maps were linearly interpolated onto a $384 \times 384 \times 3$ grid. The regions of interest were drawn by one of the authors (MCA), developmental pathologist, expert in mouse histology, specialized in brain histology, and comprised the corpus callosum (medial (mCC) and lateral (ICC)) as well as the somato-sensory cortex (CTX; layer 2/3 to 6a) according to the Allen Mouse Brain Atlas (available from: http://atlas.brain-map.org/), as shown in Figure 1 Representative rSPF and fractional anisotropy views with overlayed ROls from four mice from each group are given in Figure S2 in Supporting Information.

\subsection{6 | Statistics}

A ROI-based analysis was made, assuming that these regions are homogeneous in order to allow for a group comparison. Means and standard deviations in the averaged ROls from the three slices were used to assess the sensitivity of each parameter. A Shapiro-Wilk test was performed on each group and each parameter to test for the normality of the distributions. The mean difference between the two groups of each parameter were tested using either a bilateral Student or a bilateral Welsh test, depending on the result of a Fisher test. Additionally, Spearman correlations in both corpus callosum subregions as well as in the cortex were estimated between MRI and histological metrics. This analysis involved three distinct groups: joint control and cuprizone groups, and separated control and cuprizone groups. Adjusted p-values of the correlations were computed using Holm-Bonferroni's correction in each group.

\section{3 | RESULTS}

Representative quantitative maps selected from a mouse in each group are shown in Figure 3 The impact of the cuprizone diet on corpus callosum myelination is straightforward as shown by the decrease of the MBP fluorescent signal in treated mice.

Numerical mean and sensitivity values of the two groups computed from the various regions are presented in Table 1 Every metric followed a normal distribution according to the Shapiro-Wilk test ( $>0.05$ ), and showed systematically a significant mean difference between the two groups in the corpus callosum regions ( $p<0.05)$. rSPF, $\lambda_{\perp}$ and MWF were the only scores that did not show a significant mean difference between the two groups in the cortical region. A mean difference was estimated between $\mathrm{mCC}$ and ICC in both groups for $\mathrm{f}$, and only in the Control group for rSPF and $\lambda_{\perp}$. MRI metrics demonstrated a fairly good sensitivity to demyelination with an absolute difference higher than $40 \%$ in the corpus callosum, while MBP fluorescent intensity decreased by $23.6 \%$ and $30.1 \%$ in the ICC and $\mathrm{mCC}$, respectively. In contrast, the difference was lower in the cortical regions with an absolute difference lower than $18.5 \%$ for $r S P F, f$, and $\lambda_{\perp}$, while MWF and MBP scores were higher than $31.8 \%$. 


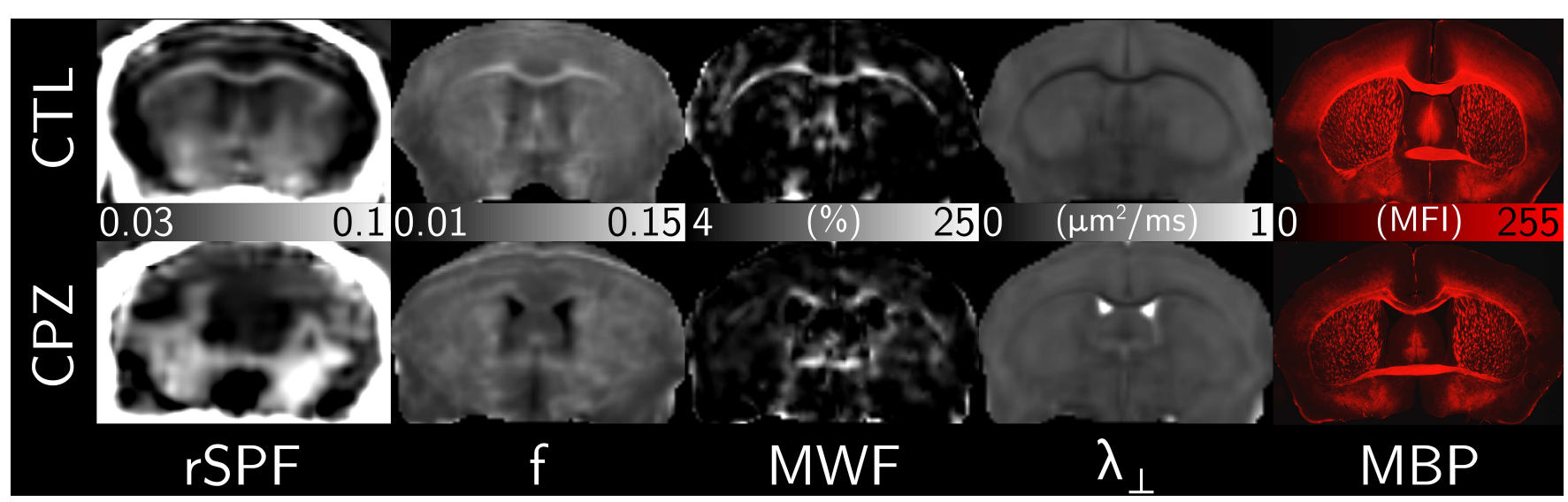

FIGURE 3 Representative quantitative maps of a Control (top) and Cuprizone (bottom) mouse. Scales are given between each map.

TABLE 1 Summary of MRI and histological metrics (mean \pm standard deviation) in the selected regions of interest, as well as the respective sensitivity (percent difference of the mean of a metric) induced by the cuprizone diet. All metrics follow a normal distribution according to the Shapiro-Wilk test $(p>0.05)$.

\begin{tabular}{|c|c|c|c|c|c|c|}
\hline Metric & \multicolumn{3}{|c|}{ Medial corpus callosum (mCC) } & \multicolumn{3}{|c|}{ Lateral corpus callosum (ICC) } \\
\hline$f$ & $0.081 \pm 0.008 x$ & $0.041 \pm 0.006 x$ & $-49.2 \%$ & $0.092 \pm 0.007$ & $0.055 \pm 0.010$ & $-40.6 \%$ \\
\hline$\lambda_{\perp}\left(\mu \mathrm{m}^{2} / \mathrm{ms}\right)$ & $0.140 \pm 0.014^{\alpha, \chi}$ & $0.227 \pm 0.033$ & $+62.5 \%$ & $0.157 \pm 0.012$ & $0.240 \pm 0.023$ & $+53.9 \%$ \\
\hline MWF (\%) & $12.72 \pm 3.09$ & $5.20 \pm 2.86$ & $-59.1 \%$ & $14.55 \pm 2.09$ & $5.83 \pm 2.94$ & $-60.3 \%$ \\
\hline
\end{tabular}

\begin{tabular}{lccc}
\hline & \multicolumn{3}{c}{ Cortex (CTX) } \\
\cline { 2 - 4 } Metric & Control & Cuprizone & Sensitivity \\
\hline rSPF & $0.050 \pm 0.009^{\dagger}$ & $0.042 \pm 0.011$ & $-15.2 \%$ \\
$\mathrm{f}$ & $0.069 \pm 0.005$ & $0.056 \pm 0.003$ & $-18.5 \%$ \\
$\lambda_{\perp}\left(\mu \mathrm{m}^{2} / \mathrm{ms}\right)$ & $0.288 \pm 0.013^{\dagger}$ & $0.308 \pm 0.024$ & $+7.3 \%$ \\
$\mathrm{MWF}(\%)$ & $2.32 \pm 1.13^{\dagger}$ & $1.58 \pm 1.21$ & $-31.8 \%$ \\
$\mathrm{MBP}(\mathrm{MFI})$ & $93.7 \pm 9.5$ & $45.5 \pm 9.6$ & $-52.1 \%$ \\
\hline
\end{tabular}

$\alpha$ : unequal variances between Control and Cuprizone groups (Fisher test; $\mathrm{p}<0.05$ )

$\chi$ : significant mean difference between ICC and mCC within the same group (Student or Welsh test; $\mathrm{p}<0.05$ )

$\dagger$ : non-significant mean difference between both groups (Student test; $p>0.05$ )

Spearman correlations between MRI metrics and MBP for each regions are shown in Table 2 along with the corresponding scatter plots in Figure 4 The macromolecular proton fraction (MPF), MWF and rSPF demonstrated significant correlations to demyelination assessed by immunohistology in both corpus callosum subregions for the joint group $(|\rho|>0.68)$, while radial diffusivity was found to be significant exclusively in the medial subregion. In the cortex, MPF was the sole metric that presented a significant correlation $(\rho=0.89)$ in the joint group. No metric demonstrated a significant correlation to myelination for both separated groups.

\section{4 | DISCUSSION}

The objective of this study was to verify whether the variation of quantitative MRI metrics following demyelination at the level of large structures (i.e., the whole corpus callosum and large cortical areas) mirrored the histological demyelination. Quantitative parameters from various protocols 
TABLE 2 Summary of the Spearman correlations for rSPF, $f, \lambda_{\perp}$ and MWF versus MBP in the corpus callosum subregions and in the cortex. Spearman correlation coefficients are reported as $\{C T L \cup C P Z-C T L / C P Z\}$.

\begin{tabular}{ccccc}
\hline MBP vs. & rSPF & $f$ & $\lambda_{\perp}$ & MWF \\
\hline Medial CC & $0.86^{*}-0.43 / 0.50$ & $0.87^{*}-0.36 / 0.64$ & $-0.70^{*}-0.38 /-0.07$ & $0.68^{*}--0.62 / 0.21$ \\
Lateral CC & $0.76^{*}-0.17 / 0.04$ & $0.91^{*}-0.55 / 0.82$ & $-0.63-0.40 / 0.14$ & $0.69^{*}--0.05 /-0.07$ \\
CTX & $0.52-0.69 /-0.03$ & $0.89^{*}-0.86 / 0.25$ & $-0.21-0.45 / 0.79$ & $0.20-0.12 /-0.64$
\end{tabular}

*: significant correlations ( $\left.p_{\text {corr }}<0.05\right)$

\section{Medial Corpus Callosum}
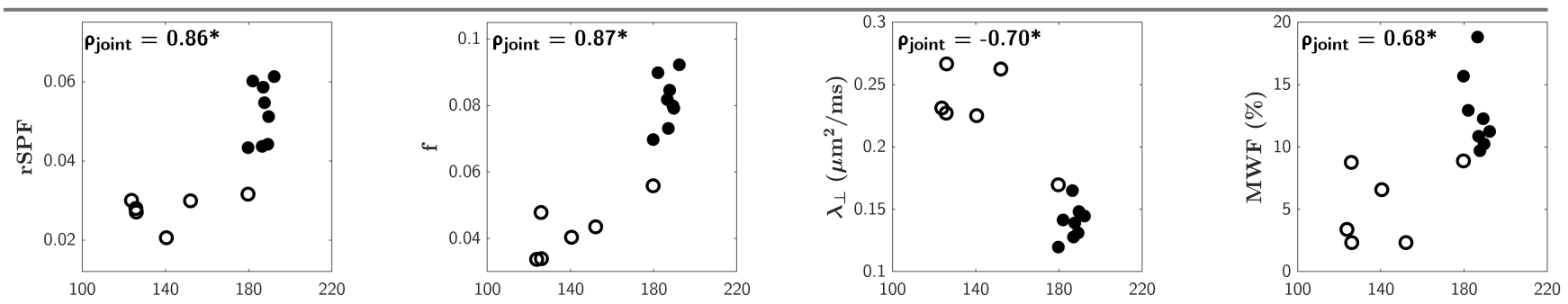

Lateral Corpus Callosum
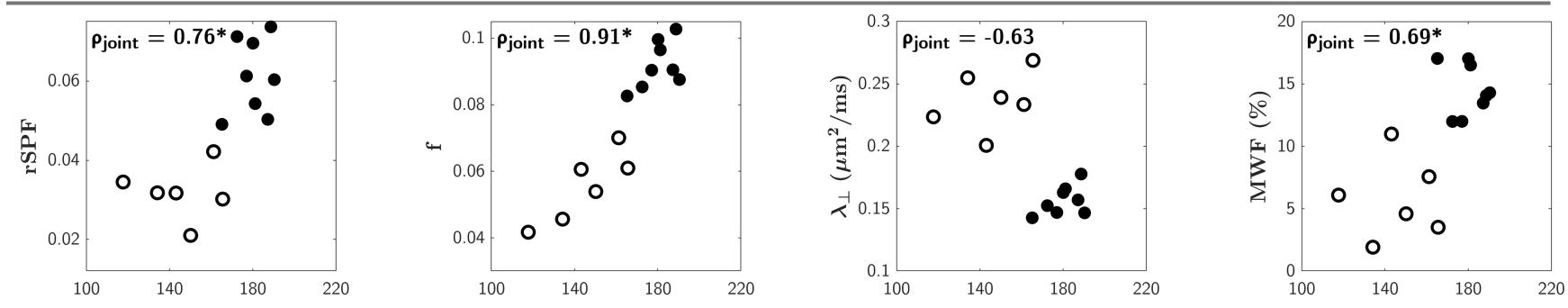

\section{Cortex}
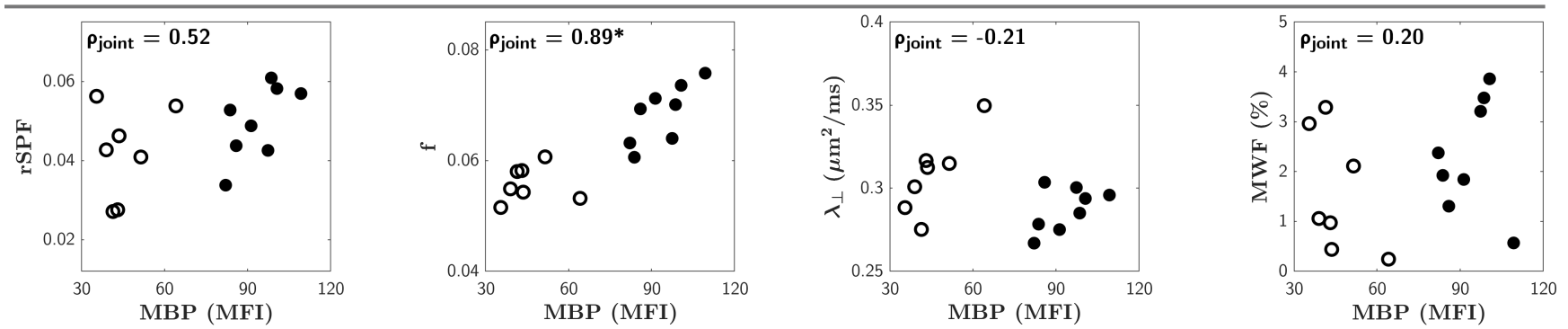

FIGURE 4 Scatter plots of MBP mean fluorescent intensity versus rSPF (first column), $\mathrm{f}$ (second column), MWF (third column) and $\lambda_{\perp}$ (fourth column) in the medial corpus callosum (top row), lateral corpus callosum (middle row) and cortex (bottom row). Control and cuprizone data are depicted in filled and open circles, respectively. Spearman correlation coefficients $(\rho)$ are reported from the joined groups, where "*" denotes significant scores.

were investigated ex vivo on mice brains, using two groups of mice, one healthy and one demyelinated by the myelin toxicant cuprizone. We demonstrated expected high sensitivities to demyelination of various quantitative MRI metrics, including the macromolecular proton fraction, myelin water fraction, relative semi-solid proton fraction and radial diffusivity. The results were compared to the mean fluorescence intensity of the Myelin Basic Protein following immuno-staining of histological sections. The rSPF and MPF showed to be highly sensitive to demyelination, and shared a strong affinity to the MBP MFI, which is consistent with the biophysical model2425. Among the investigated MRI metrics and in terms of demyelination process assessed by MBP immuno-staining, the MPF remains the most indicative metric, followed by rSPF. MWF and radial 
diffusivity, respective markers of myelin sheath and axonal integrity, showed reduced significance in correlations; this may however be attributed to the histology marker, highly specific to the myelin macromolecular content.

The results regarding the high correlations of $\mathrm{SPF}$ and MPF to the MBP MFI support the hypothesis of a direct acquisition of signals arising from the semi-solid matrix of myelinin UTE, and also further validate the quantitative magnetization transfer model since proteins constitute a major media for the phenomenon. Nonetheless, the lower correlations in the cortex of rSPF versus $f$ and MBP may be attributed to a different signal source between these modalities: both techniques rely upon the presence of a short- $\mathrm{T}_{2}$ pool, although the respective contrasts are generated differently. The qMT method requires a media for the magnetization transfer mechanisms (e.g. labile protons and solvation layer typically found in proteins), whereas UTE signals supposedly arise from the entire available ${ }^{1} \mathrm{H}$ spectrum (i.e. static and labile protons) 2425 ! Under the assumption of a unique $\mathrm{T}_{2}$ value of about $10 \mu \mathrm{s}$ regarding the restricted pool in qMTI, the Diff-UTE sequence may only acquire a minor part of the macromolecular content responsible for the magnetization transfer phenomenon under our sequence parameters ( $T E / \tau_{2}=10 / 10 \mu \mathrm{s}$ ). Consequently, both metrics could be complementary since the majority of the Diff-UTE contrast most likely takes its origin in the more slowly relaxing macromolecular spectra $\left(\mathrm{T}_{2} \in[10 ; 1000] \mu \mathrm{s}\right)$.

The signal normalization in Diff-UTE assumes an invariant $T_{1}$ value of the biological structures of interest although these structures might be changed by the cuprizone diet, implying $T_{1}$ modifications of the semi-solid part of the myelin. A proton density analysis would provide a more precise and absolute measurement of the actual state of the myelin structure; unfortunately, $T_{1}$ relaxometry experiments of short- $T_{2}$ components remain challenging and require further adjustments as well as an increased acquisition time.

In the separated groups (either Control or Cuprizone), no significant correlation was found between MRI and histology metrics, nor between MRI metrics. This observation may stem from the limited amount of samples in each group. This also emphasizes the necessity of $\mathrm{p}$-value correction in such analyses since high scores with counterintuitive polarity (e.g. $\rho=-0.86$ between rSPF and MPF in the Cortex in the Cuprizone group; see Table T1 in SI) are most likely spurious.

In this study, the preferential SNR situation $\left(\approx 100\right.$ on $\mathrm{MT}_{0}$ prior to the denoising step) allowed to estimate the qMT parameters through the Sled and Pike's RP model. Among the similar investigations at $7 \mathrm{~T}$ over a murine model involving a group of mice demyelinated by a cuprizone diet, Thiessen et al. ${ }^{4}$ and Turati et al. ${ }^{33}$ employed Ramani's model for parametric estimations $\sqrt{45}$. However, it has been demonstrated by Cercignani et al. 11 that this method leads to an underestimation of $\mathrm{f}$, contrary to Sled and Pike's RP model, in spite of being more robust in case of a low SNR. In addition, Ramani's model is sensitive to both the RF duty cycle and the excitation flip angle introducing a bias due to $T_{1}$-weighting. Also, it should be noticed that in both studies, no account of potential $B_{1}$ deviations was made, inherently biasing the resulting $f$ in case of $B_{1}$ field inhomogeneities or miscalibration. Nonetheless, the mean $\mathrm{f}$ value estimated in the corpus callosum of the control group in our study was still lower than the nominal value of about 0.10 found in healthy mice and humans ${ }^{446}$, and yet similar to those of Ou et al. ${ }^{47}$ ( $\mathrm{f} \approx 0.09$ ) who employed a selective inversion recovery fast-spin-echo sequence. Khodanovich et al. ${ }^{5}$ estimated higher values in healthy mice $(\mathrm{f} \approx 0.13$ in the $\mathrm{CC})$ at $11.7 \mathrm{~T}$ using Yarnykh's single-point $f$ mapping 4148 .

Myelin water imaging remains a standard method for white matter monitoring 24950. However, tissue fixation protocols deeply modify the multi-compartmental behavior of cerebral tissues, leading to an observable mono-compartmentalization of water $\mathrm{T}_{2}$ distribution in white matter, as reported by Thiessen et al. 4 who employed a 0.5\%-glutaraldehyde/2\%-PFA fixation. Wood et al. 22 estimated MWF using a fixation protocol similar to ours, and by means of the mcDESPOT model ${ }^{18}$. To our knowledge, the present preclinical study is the first one performing a MWF NNLS-based analysis using a multi spin-echo sequence in an ex vivo cuprizone model of demyelination. MWF values are consistent with that found in literature in healthy human white matter ${ }^{5152}$, but also remains lower than those estimated in mcDESPOT 22 (MWF $\approx 20 \%$ ). Nonetheless, the difference between these estimations may not only arise from the arbitrary MWF-T 2 cutoff in the NNLS analysis, but also from the absence of consideration of chemical exchange between the different compartments which is an important dynamic aspect of water molecules evolving within the microstructure 2220 .

To compensate for a lack of signal-to-noise ratio (SNR) in brain tissues in Diff-UTE, an anisotropic voxel geometry was preferred in the slice direction. In addition, the manufacturer native 3D-UTE sequence does not allow for any field of view re-positioning, and heads placement remained challenging for reproducibility purpose since it was therefore made by mechanical shifting of the cell inside the bore. Both features may lead to increased partial volume effects in regions of interest, especially in the thin white matter area of the mouse brain, although the linear interpolation prior to ROI delineations aimed at diminishing such effects. The use of a 23-mm volume transceiver coil was initially chosen to provide an homogeneous $B_{1}$ profile in the slab of interest, as the Diff-UTE suppression scheme is sensitive to such inhomogeneities (see Supporting Information). The use of more sensitive signal reception systems in pair with a transmitter volume coil may represents an alternative to address these issues.

In spite of a high correlation with to macromolecular content metrics (MBP and f; see Table T1 in SI) in the corpus callosum, rSPF correlates moderately with these in the cortex. As can be noticed in Figure 3 on the rSPF maps, a dominant signal arises from the skull. Hence, estimations from ROIs placed in the cortex may suffer from contaminations inherent to Gibbs artifacts and blurry effects caused by the relatively low receiver bandwidth in Diff-UTE and short $\mathrm{T}_{2}$ values in the cortical bone. 
In this study, we made use of the Diff-UTE sequence for a first assessment of the sensitivity of a UTE sequence to demyelination in a preclinical environment. This sequence was preferred to the commonly-used IR-UTE ${ }^{26}$ since it allows for higher short- $\mathrm{T}_{2}$ signal generation 31 . From a demyelination point of view, the rSPF scores demonstrated an interesting sensitivity, as well as high correlations to macromolecular content metrics. However, as any long- $\mathrm{T}_{2}$ suppressed UTE method, the sequence requires optimum hardware capabilities (e.g. RF peak power for short- $\mathrm{T}_{2}$ signal maximization), but also strong gradients for diffusivity purpose and a reasonable $\mathrm{B}_{1}^{+}$profile (see Figure S1 in Supporting Information): these constraints may represent an issue toward the applicability over standard high-field clinical systems. Although, UTE acquisitions on such systems may be highly beneficial for different aspects. For example, the employed radial acquisition pattern represents a permissive feature for sequence acceleration (undersampling and sparse reconstructions ${ }^{5354}$ ), especially in high signal-to-noise ratio conditions with increased voxel sizes. It is also advantageous for its motion insensitivity intrinsic ability 55 .

The precise spectrum composition in the white matter following a cuprizone demyelination remains to be established as white matter microstructure following demyelination is changed. The changes comprise patchy absence of myelin sheath, presence of intra/extracellular myelin debris, increased number and size of reactive cells (microglia, astrocytes) and oligodendrocyte progenitors proliferation 56 . These alterations have an impact over dipolar interactions experienced by protons carried by semi-solid structures, hence lowering the mechanisms that mainly influence the short$\mathrm{T}_{2}$ phenomenon. The $\mathrm{T}_{2}$-spectrum of the semi-solid part of myelin will suffer from such structural modifications, and short relaxing components may shift toward higher values. However, estimating the actual state of this spectrum remains complex in UTE sequences due to the low SNR in myelinated structures and other filtering and weighing effects (e.g. low TR and $\mathrm{T}_{2}$ relaxation during excitation). In order to reduce such undesirable effects, one may for example increase the repetition time, although leading to prohibitively long scan times. A precise exploration of the biological content using an extended panel of biomarkers such as additional MRI quantitative parameters, electron microscopy and immuno-histology for the different cell types and oligodendrocytes differentiation stages 5758 will allow for a better characterization of the white matter microstructure and myelin state.

Leprince et al. .59 demonstrated variations of a mono-component $\mathrm{T}_{2}$ as a function of the post-fixation conservation duration. Chen et al. 50 observed a myelin sheath loosening and cell shrinkage, simultaneously modifying the respective size of the various water compartments, and hence MWF. Sheath loosening most likely leads to a degraded mechanism responsible for $\mathrm{T}_{2}$ shortening of trapped water in myelin sheaths. As a result, the $\mathrm{T}_{2}$ distribution of myelin water may shift and distort depending on the type of employed fixation, and the optimal $\mathrm{T}_{2}$ cutoff in NNLS-analyses has to be modulated as such. The inability of Thiessen et al. ${ }^{4}$ to perform $\mathrm{MWI}$ is most likely due to such phenomena. Further investigations have yet to be led in order to determine the optimal $\mathrm{T}_{2}$-cutoff as well as a robust fixation protocol for ex vivo myelin imaging.

In the present study, a single protein was marked for myelin quantification by histology, showing a high correlation with putative MRI markers indicative of macromolecular content ( $f$ and rSPF). Ideally, myelin quantification should be confronted to electron microscopy. Unfortunately, the fixation protocol imposes the use of glutaraldehyde and PFA, leading to a $\mathrm{T}_{2}$ shortening and mono-compartmentalization in soft tissues, preventing $\mathrm{MWI}$ analyses ${ }^{4}$. In addition, it has been shown that Diff-UTE efficiency for long- $\mathrm{T}_{2}$ signal suppression is reduced if the diffusive species were to present too short $\mathrm{T}_{2}$ values 31 .

The objective of the histology study was to verify whether the variation of MRI parameters following demyelination at the level of large structures (e.g. the whole corpus callosum or large cortical areas) mirrored the histological demyelination. Histology is a versatile technique as its in-plane resolution streches from nanometer to millimeter, whether its in-slice resolution may go from a few tens of nanometers to a few hundreds micrometers. In this study, we used low in-slice MRI partition; accordingly, histological technique was adapted to favor high fluorescence yield by the use of thick histological sections. Other histological techniques can be used for myelin quantification, namely myelin scoring following LFB staining 560 . Although easy to perform, the scoring is highly subjective and dependent upon the observer. Moreover, tissue processing for LFB staining requires prolonged fixation in order to perform paraffin embedding and $5 \mu \mathrm{m}$-thick tissue sectioning. On one hand, prolonged fixation and technical procedure for paraffin embedding (i.e. dehydration) distort protein architecture and thus the relevance of histology toward quantitative in vivo MRI; on the other hand, $5 \mu \mathrm{m}$-thick tissue sections are poor references for volumes even if their number is multiplied. In order to diminish these biases, short fixation times were used followed by residual free fixative removal with isotonic solution. Sectioning were performed with a vibratome prior to immuno-staining, a method which does not require tissue dehydration and allows sectioning between 40 and $200 \mu \mathrm{m}$. 5 weeks $0.2 \%$ cuprizone administration induces global, but not total corpus callosum demyelination: on coronal sections, patches of total demyelination are intermingled with areas displaying intact myelin 43 . Moreover, caudal and rostral corpus callosum are not equally sensitive to cuprizone demyelination which induces a rostro-caudal demyelination gradient ${ }^{44}$ at 5 weeks. In this last study, authors considered ROls of $200 \mu \mathrm{m}$ in length along the corpus callosum and showed that within the millimeter preceding bregma, the demyelination is lower than in the second millimeter following bregma. The authors examined, when available, two to three $20 \mu \mathrm{m}$-thick sections (thus representing 40 to $60 \mu \mathrm{m}$ ) in 5 demyelinated mice and reported a mean demyelination score. In our study, we examined one $60 \mu \mathrm{m}$-thick rostral section within the 0.5 millimeters anterior to bregma and one $60 \mu \mathrm{m}$-thick caudal section within the second millimeter posterior to bregma, thus placing our histological references according to published references.

The parameters sensitivity analysis between the two groups assesses the mean difference of a metric following demyelination, assuming homogeneous groups. Unfortunately, estimations demonstrated a dispersion depending on the brain tissue state prior to acquisitions (e.g. conservation 
and myelin loss) as well as parametric estimations. Thus, such analysis is moderately informative about the superiority of a metric over another to quantify myelin changes as a function of demyelination. Increasing the number of sample examined and minimizing intra-group experimental variability should allow for more precise interpretations.

In this study, the analyses were performed by comparing the mean values of voxels comprised within structures of interest in each mouse. In spite of particular attentions regarding ROI delineations, inter-individual variabilities may propagate and bias estimations. To reduce these issues, an atlas could be constituted in order to perform a more rigorous voxel-based analysis 6137 . However in our case, anatomical variabilities (e.g. variable ventricles dilation in the cuprizone mice) and high voxels anisotropy make atlases constitution challenging. Furthermore, the particular contrast generated in Diff-UTE prevents from automatic registration onto soft-tissues contrasted images.

Due to the multiple quantitative protocols resulting in a prohibitively long total acquisition duration, ex vivo experiments were preferred. Tissue states and experimental conditions (e.g. sample temperature, tissue permeability to free water and ratio of the various intra/extracellular spaces) were very different from in vivo conditions 626364 . Potential variabilities in the statistics based on quantitative metrics may therefore occur in an in vivo study. Nonetheless, the present study shows encouraging results and represents a first step toward in vivo validation using an updated Diff-UTE protocol.

\section{5 | CONCLUSIONS}

We have assessed the sensitivities and correlations of various quantitative MRI parameters to histological immuno-marking of the MBP in ex vivo healthy and demyelinated mice brains. The macromolecular proton fraction from qMTI presented the highest correlation in the corpus callosum, followed by the rSPF from Diff-UTE. Alternatively, radial diffusivity from DTI and myelin water fraction from MWI demonstrated high sensitivities, but moderate correlations, which is somewhat inherent to the underlying signal source from these two metrics. This emphasizes the need of comprehensive quantitative protocols to better characterize complex biological tissues from the central nervous system.

\section{ACKNOWLEDGMENTS}

The authors thank Dr. Laura Harsan for the careful manuscript reviews, and Julien Graff for technical support.

\section{SUPPORTING INFORMATION}

The following supporting information is available as part of the online article:

Figure S1. Simulated long- $\mathrm{T}_{2}$ signals (a) as a function of $\mathrm{B}_{1}$ deviation and preparation flip angle $\alpha_{1}$ for optimized $\alpha_{2}$, and (b) as a function of $\mathrm{B}_{1}$ deviation and readout flip angle $\alpha_{2}$ for $\alpha_{1}=90^{\circ}$ (the white dashed line corresponding to the optimal $\alpha_{2}$ for this parameter set). Simulation parameters: long relaxing component $\mathrm{T}_{1}^{\mathrm{L}} / \mathrm{T}_{2}^{\mathrm{L}}=1000 / 100 \mathrm{~ms}$ and $\mathrm{D}=1.00 \mu \mathrm{m}^{2} / \mathrm{ms}$, short relaxing component $\mathrm{T}_{1}^{\mathrm{S}} / \mathrm{T}_{2}^{\mathrm{S}}=300 / 0.315 \mathrm{~ms}, \tau_{1} / \tau_{2}=$ $1 / 0.01 \mathrm{~ms}, \mathrm{TR}_{1} / \mathrm{TR}_{2}=5 / 25 \mathrm{~ms}$. Signal nulling occurrences $\left(\mathrm{M}_{\mathrm{z}}^{\mathrm{L}} / \mathrm{M}_{0}=\mathrm{S}^{\mathrm{L}}<0.05 \% \mathrm{M}_{0}\right)$ are depicted in the blue bands. Simulations were performed using the Extended Phase Graph algorithm.

Figure S2. Exemplary fractional anisotropy and rSPF maps of the three axial views from four mice from the Control (CTL; top) and Cuprizone (CPZ; bottom) groups.

Table T1. Summary of the Spearman correlations between each MRI metrics rSPF, $\mathrm{f}, \lambda_{\perp}$ and MWF in the corpus callosum subregions and in the cortex. Spearman correlation coefficients are reported as $\{C T L \cup C P Z-C T L / C P Z\}$

\section{References}

1. Basser P, Mattiello J, Lebihan D. Estimation of the Effective Self-Diffusion Tensor from the NMR Spin Echo. Journal of Magnetic Resonance, Series B 1994; 103: 247-254. doi: 10.1006/jmrb.1994.1037

2. Whittall KP, MacKay AL. Quantitative interpretation of NMR relaxation data. Journal of Magnetic Resonance (1969) 1989; 84(1): 134-152. doi: 10.1016/0022-2364(89)90011-5

3. Henkelman RM, Huang X, Xiang QS, Stanisz GJ, Swanson SD, Bronskill MJ. Quantitative interpretation of magnetization transfer. Magnetic Resonance in Medicine 1993; 29(6): 759-766. doi: 10.1002/mrm.1910290607 
4. Thiessen JD, Zhang Y, Zhang H, et al. Quantitative MRI and ultrastructural examination of the cuprizone mouse model of demyelination. NMR in Biomedicine 2013; 26(11): 1562-1581. doi: 10.1002/nbm.2992

5. Khodanovich MY, Sorokina IV, Glazacheva VY, et al. Histological validation of fast macromolecular proton fraction mapping as a quantitative myelin imaging method in the cuprizone demyelination model. Scientific Reports 2017; 7(March): 46686. doi: 10.1038/srep46686

6. Enzinger C, Barkhof F, Ciccarelli O, et al. Nonconventional MRI and microstructural cerebral changes in multiple sclerosis. Nature Reviews Neurology 2015; 11(12): 676-686. doi: 10.1038/nrneurol.2015.194

7. Song SK, Yoshino J, Le TQ, et al. Demyelination increases radial diffusivity in corpus callosum of mouse brain. Neurolmage 2005; 26(1): 132-140. doi: 10.1016/j.neuroimage.2005.01.028

8. Harsan LA, Steibel J, Zaremba A, et al. Recovery from Chronic Demyelination by Thyroid Hormone Therapy: Myelinogenesis Induction and Assessment by Diffusion Tensor Magnetic Resonance Imaging. Journal of Neuroscience 2008; 28(52): 14189-14201. doi: 10.1523/JNEUROSCI.4453-08.2008

9. Hüppi PS, Dubois J. Diffusion tensor imaging of brain development. Seminars in Fetal and Neonatal Medicine 2006; 11(6): 489-497. doi: 10.1016/j.siny.2006.07.006

10. Sled JG, Pike GB. Quantitative imaging of magnetization transfer exchange and relaxation properties in vivo using MRI. Magnetic Resonance in Medicine 2001; 46(5): 923-931. doi: 10.1002/mrm.1278

11. Cercignani M, Barker GJ. A comparison between equations describing in vivo MT: The effects of noise and sequence parameters. Journal of Magnetic Resonance 2008; 191(2): 171-183. doi: 10.1016/j.jmr.2007.12.012

12. Mackay A, Whittall K, Adler J, Li D, Paty D, Graeb D. In vivo visualization of myelin water in brain by magnetic resonance. Magnetic Resonance in Medicine 1994; 31(6): 673-677. doi: 10.1002/mrm.1910310614

13. Prasloski T, Mädler B, Xiang QS, MacKay A, Jones C. Applications of stimulated echo correction to multicomponent T2 analysis. Magnetic Resonance in Medicine 2012; 67(6): 1803-1814. doi: 10.1002/mrm.23157

14. Layton KJ, Morelande M, Wright D, Farrell PM, Moran B, Johnston La. Modelling and Estimation of Multicomponent T2 Distributions. IEEE Transactions on Medical Imaging 2013; 32(8): 1423-1434. doi: 10.1109/TMI.2013.2257830

15. Nam Y, Lee J, Hwang D, Kim DH. Improved estimation of myelin water fraction using complex model fitting. Neurolmage 2015; $116: 214-221$. doi: 10.1016/j.neuroimage.2015.03.081

16. Lee H, Nam Y, Lee HJ, Hsu JJ, Henry RG, Kim DH. Improved three-dimensional multi-echo gradient echo based myelin water fraction mapping with phase related artifact correction. Neurolmage 2018; 169(November 2017): 1-10. doi: 10.1016/j.neuroimage.2017.11.058

17. Thapaliya K, Vegh V, Bollmann S, Barth M. Assessment of microstructural signal compartments across the corpus callosum using multi-echo gradient recalled echo at 7 T. Neurolmage 2018; 182(September): 407-416. doi: 10.1016/j.neuroimage.2017.11.029

18. Deoni SCL, Matthews L, Kolind SH. One component? Two components? Three? The effect of including a nonexchanging "free" water component in multicomponent driven equilibrium single pulse observation of T 1 and T 2. Magnetic Resonance in Medicine 2013; 70(1): 147-154. doi: $10.1002 / \mathrm{mrm} .24429$

19. Zhang J, Kolind SH, Laule C, MacKay AL. Comparison of myelin water fraction from multiecho T2 decay curve and steady-state methods. Magnetic Resonance in Medicine 2015; 73(1): 223-232. doi: 10.1002/mrm.25125

20. Bouhrara M, Reiter DA, Celik H, Fishbein KW, Kijowski R, Spencer RG. Analysis of mcDESPOT- and CPMG-derived parameter estimates for two-component nonexchanging systems. Magnetic Resonance in Medicine 2016; 75(6): 2406-2420. doi: 10.1002/mrm.25801

21. Lankford CL, Does MD. On the inherent precision of mcDESPOT. Magnetic Resonance in Medicine 2013; 69(1): 127-136. doi: 10.1002/mrm.24241

22. Wood TC, Simmons C, Hurley SA, et al. Whole-brain ex-vivo quantitative MRI of the cuprizone mouse model. PeerJ 2016; 4: e2632. doi: 10.7717/peerj.2632 
23. Bydder GM, Fullerton GD, Young IR. MRI of tissues with short T2s and T2*s . 2012

24. Horch RA, Gore JC, Does MD. Origins of the ultrashort-T2 $1 \mathrm{H}$ NMR signals in myelinated nerve: A direct measure of myelin content?. Magnetic Resonance in Medicine 2011; 66(1): 24-31. doi: 10.1002/mrm.22980

25. Wilhelm MJ, Ong HH, Wehrli SL, et al. Direct magnetic resonance detection of myelin and prospects for quantitative imaging of myelin density. Proceedings of the National Academy of Sciences 2012; 109(24): 9605-9610. doi: 10.1073/pnas.1115107109

26. Du J, Ma G, Li S, et al. Ultrashort echo time (UTE) magnetic resonance imaging of the short T2 components in white matter of the brain using a clinical 3T scanner.. Neurolmage 2014; 87: 32-41. doi: 10.1016/j.neuroimage.2013.10.053

27. Du J, Sheth V, He Q, et al. Measurement of T1 of the Ultrashort T2* Components in White Matter of the Brain at 3T. PLoS ONE 2014; 9(8): e103296. doi: 10.1371/journal.pone.0103296

28. Sheth V, Shao H, Chen J, et al. Magnetic resonance imaging of myelin using ultrashort Echo time (UTE) pulse sequences: Phantom, specimen, volunteer and multiple sclerosis patient studies. Neurolmage 2016; 136: 37-44. doi: 10.1016/j.neuroimage.2016.05.012

29. Chen J, Chang EY, Carl M, et al. Measurement of bound and pore water T1 relaxation times in cortical bone using three-dimensional ultrashort echo time cones sequences. Magnetic Resonance in Medicine 2017; 77(6): 2136-2145. doi: 10.1002/mrm.26292

30. Larson PEZ, Conolly SM, Pauly JM, Nishimura DG. Using adiabatic inversion pulses for long-T2 suppression in ultrashort echo time (UTE) imaging. Magnetic Resonance in Medicine 2007; 58(5): 952-961. doi: 10.1002/mrm.21341

31. Soustelle L, Lamy J, Rousseau F, Armspach JP, Loureiro de Sousa P. A diffusion-based method for long- T 2 suppression in steady state sequences: Validation and application for 3D-UTE imaging. Magnetic Resonance in Medicine 2018; 80(2): 548-559. doi: 10.1002/mrm.27057

32. Soustelle L, Loureiro de Sousa P, Lamy J, Santin DM, Rousseau F, Armspach J. Myelin and Cortical Bone Short-T2 Quantification Using Saturation and Diffusion-Based Long-T2 Suppression in a Steady-State 3D-UTE Sequence. In: ; 2017; Honolulu, Hawaii, USA: 4030.

33. Turati L, Moscatelli M, Mastropietro A, et al. In vivo quantitative magnetization transfer imaging correlates with histology during de- and remyelination in cuprizone-treated mice. NMR in Biomedicine 2015; 28(3): 327-337. doi: 10.1002/nbm.3253

34. Yarnykh VL. Actual flip-angle imaging in the pulsed steady state: A method for rapid three-dimensional mapping of the transmitted radiofrequency field. Magnetic Resonance in Medicine 2007; 57(1): 192-200. doi: 10.1002/mrm.21120

35. Zur Y, Wood ML, Neuringer LJ. Spoiling of transverse magnetization in steady-state sequences. Magnetic Resonance in Medicine 1991; 21: 251-263. doi: $10.1002 / \mathrm{mrm} .1910210210$

36. Nehrke K. On the steady-state properties of actual flip angle imaging (AFI). Magnetic Resonance in Medicine 2009; 61(1): 84-92. doi: 10.1002/mrm.21592

37. Avants BB, Tustison NJ, Song G, Cook PA, Klein A, Gee JC. A reproducible evaluation of ANTs similarity metric performance in brain image registration. Neurolmage 2011; 54(3): 2033-2044. doi: 10.1016/j.neuroimage.2010.09.025

38. Avants B, Tustison N, Song G. Advanced Normalization Tools (ANTS). Insight J. 2 2009: 1-35.

39. Maggioni M, Katkovnik V, Egiazarian K, Foi A. Nonlocal Transform-Domain Filter for Volumetric Data Denoising and Reconstruction. IEEE Transactions on Image Processing 2013; 22(1): 119-133. doi: 10.1109/TIP.2012.2210725

40. Cabana JF, Gu Y, Boudreau M, et al. Quantitative magnetization transfer imaging made easy with qMTLab : Software for data simulation, analysis, and visualization. Concepts in Magnetic Resonance Part A 2015; 44A(5): 263-277. doi: 10.1002/cmr.a.21357

41. Yarnykh VL. Fast macromolecular proton fraction mapping from a single off-resonance magnetization transfer measurement. Magnetic Resonance in Medicine 2012; 68(1): 166-178. doi: 10.1002/mrm.23224

42. Hwang D, Du YP. Improved myelin water quantification using spatially regularized non-negative least squares algorithm. Journal of Magnetic Resonance Imaging 2009; 30(1): 203-208. doi: 10.1002/jmri.21783

43. Stidworthy MF, Genoud S, Suter U, Mantei N, Franklin RJM. Quantifying the Early Stages of Remyelination Following Cuprizone-induced Demyelination. Brain Pathology 2003; 13(3): 329-339. doi: 10.1111/j.1750-3639.2003.tb00032.x 
44. Steelman AJ, Thompson JP, Li J. Demyelination and remyelination in anatomically distinct regions of the corpus callosum following cuprizone intoxication. Neuroscience Research 2012; 72(1): 32-42. doi: 10.1016/j.neures.2011.10.002

45. Ramani A, Dalton C, Miller D, Tofts P, Barker G. Precise estimate of fundamental in-vivo MT parameters in human brain in clinically feasible times. Magnetic Resonance Imaging 2002; 20(10): 721-731. doi: 10.1016/S0730-725X(02)00598-2

46. Levesque IR, Giacomini PS, Narayanan S, et al. Quantitative magnetization transfer and myelin water imaging of the evolution of acute multiple sclerosis lesions. Magnetic Resonance in Medicine 2010; 63: 633-640. doi: 10.1002/mrm.22244

47. Ou X, Sun SW, Liang HF, Song SK, Gochberg DF. The MT pool size ratio and the DTI radial diffusivity may reflect the myelination in shiverer and control mice. NMR in Biomedicine 2009; 22(5): 480-487. doi: 10.1002/nbm.1358

48. Naumova AV, Akulov AE, Khodanovich MY, Yarnykh VL. High-resolution three-dimensional macromolecular proton fraction mapping for quantitative neuroanatomical imaging of the rodent brain in ultra-high magnetic fields. Neurolmage 2017; 147: 985-993. doi: 10.1016/j.neuroimage.2016.09.036

49. Laule C, Vavasour IM, Kolind SH, et al. Magnetic resonance imaging of myelin.. Neurotherapeutics : the journal of the American Society for Experimental NeuroTherapeutics 2007; 4(3): 460-84. doi: 10.1016/j.nurt.2007.05.004

50. Chen HSM, Holmes N, Liu J, Tetzlaff W, Kozlowski P. Validating myelin water imaging with transmission electron microscopy in a rat spinal cord injury model. Neurolmage 2017; 153(March): 122-130. doi: 10.1016/j.neuroimage.2017.03.065

51. Oh J, Han ET, Lee MC, Nelson SJ, Pelletier D. Multislice Brain Myelin Water Fractions at 3T in Multiple Sclerosis. Journal of Neuroimaging 2007; 17(2): 156-163. doi: 10.1111/j.1552-6569.2007.00098.x

52. MacKay A, Laule C, Vavasour I, Bjarnason T, Kolind S, Madler B. Insights into brain microstructure from the T2 distribution. Magnetic Resonance Imaging 2006; 24(4): 515-525. doi: 10.1016/j.mri.2005.12.037

53. Block KT, Uecker M, Frahm J. Undersampled radial MRI with multiple coils. Iterative image reconstruction using a total variation constraint. Magnetic Resonance in Medicine 2007; 57(6): 1086-1098. doi: 10.1002/mrm.21236

54. Lustig M, Donoho D, Pauly JM. Sparse MRI: The application of compressed sensing for rapid MR imaging. Magnetic Resonance in Medicine 2007; 58(6): 1182-1195. doi: 10.1002/mrm.21391

55. Song J, Liu Y, Gewalt SL, Cofer G, Johnson GA, Liu QH. Least-square NUFFT methods applied to 2-D and 3-D radially encoded MR image reconstruction. IEEE Transactions on Biomedical Engineering 2009; 56(4): 1134-1142. doi: 10.1109/TBME.2009.2012721

56. Matsushima GK, Morell P. The Neurotoxicant, Cuprizone, as a Model to Study Demyelination and Remyelination in the Central Nervous System. Brain Pathology 2006; 11(1): 107-116. doi: 10.1111/j.1750-3639.2001.tb00385.x

57. Bradl M, Lassmann H. Oligodendrocytes: biology and pathology. Acta Neuropathologica 2010; 119(1): 37-53. doi: 10.1007/s00401-009$0601-5$

58. Barateiro A, Fernandes A. Temporal oligodendrocyte lineage progression: In vitro models of proliferation, differentiation and myelination. Biochimica et Biophysica Acta (BBA) - Molecular Cell Research 2014; 1843(9): 1917-1929. doi: 10.1016/j.bbamcr.2014.04.018

59. Leprince Y, Schmitt B, Chaillou É, et al. Optimization of sample preparation for MRI of formaldehyde-fixed brains. In: ; $2015: 2283$.

60. Laule C, Leung E, Li DK, et al. Myelin water imaging in multiple sclerosis: quantitative correlations with histopathology. Multiple Sclerosis 2006; 12(6): 747-753. doi: 10.1177/1352458506070928

61. Goto M, Abe O, Aoki S, et al. Diffeomorphic Anatomical Registration Through Exponentiated Lie Algebra provides reduced effect of scanner for cortex volumetry with atlas-based method in healthy subjects. Neuroradiology 2013; 55(7): 869-875. doi: 10.1007/s00234-013-1193-2

62. Wu D, Xu J, McMahon MT, et al. In vivo high-resolution diffusion tensor imaging of the mouse brain. Neurolmage 2013; 83: 18-26. doi: 10.1016/j.neuroimage.2013.06.012

63. Seifert A, Umphlett M, Fowkes M, Xu J. Formalin Tissue Fixation Biases Myelin Density Measurement by Quantitative Magnetization Transfer and Myelin Water Imaging. In: ; 2018: 322. 
64. Fishbein KW, Gluzband YA, Kaku M, et al. Effects of formalin fixation and collagen cross-linking onT2 and magnetization transfer in bovine nasal cartilage. Magnetic Resonance in Medicine 2007; 57(6): 1000-1011. doi: 10.1002/mrm.21216 